\title{
Epidemiology of potential drug-drug interactions in elderly population admitted to critical care units of Peshawar, Pakistan
}

\author{
Faisal Shakeel ${ }^{1 *}$ D, Muhammad Aamir ${ }^{1}$, Ahmad Farooq Khan², Tayyiba Nader Khan ${ }^{3}$ and Samiullah Khan ${ }^{4}$
}

\begin{abstract}
Background: Aging population, is a reality in many countries because of improvement in the health care, patient safety and other supplemental factors. Pharmacotherapy in this population must be evaluated due to their higher susceptibility to adverse drug outcomes, like potential drug-drug interactions (PDDIs). Research in this regard is limited particularly in developing countries. The aim of the study was to evaluate the prevalence and associated factors in this population.

Methods: The multicentered study evaluated the prevalence of potential drug-drug interactions and associated factors in elderly population at critical care units in Peshawar, Pakistan. Potential drug-drug interactions were evaluated using Micromedex DrugReax, while statistical analysis was performed using SPSS.

Results: A total of $70.17 \%$ elderly patients were observed to have at least one PDDI. A significant association was observed between presence of PDDls and number of prescribed drugs, duration of stay and age $(p<0.05)$. A total of 3019 PDDls were observed, attributing to 225 drug pairs. Prevalent PDDls were of moderate severity, good documentation and pharmacodynamic in nature. One-way ANOVA revealed a significant difference in the means of PDDls between Northwest general hospital and the rest of the hospitals. Moreover, there was a significant difference in the means of PDDIs of CCU and SU with rest of the units.

Conclusion: The prevalence of PDDls was observed to be high in elderly population which can be managed by avoiding or managing a limited number of drug combinations. Such studies are necessary to evaluate the risks of these PDDIs in a population which is already physiologically compromised.
\end{abstract}

Keywords: Geriatrics, Potential drug-drug interactions, Epidemiology

\section{Background}

An outcome of interrelated developmental achievements is an ageing population. Improvement in healthcare is a major factor for increasing life expectancy, along with supplemental factors like improved nutrition, education, income and sanitation [1]. Patient safety has gained much attention in recent years by health care providers, further incrementing the age of the population. Pharmacotherapy has aided in improving health of the patients, however, it has also led to a rise in adverse drug events. One such adverse event is drug-drug interactions [2].

\footnotetext{
* Correspondence: Faisalshakeel1@gmail.com

'Department of Pharmacy, Sarhad University of Science and IT, Peshawar, Pakistan Full list of author information is available at the end of the article
}

Elderly population, known as geriatrics, face many health issues due to the natural process of ageing, beyond the control of humans. Treating multi-morbidities in geriatrics with drugs is complex and leads to the expression of adverse drug events. This along with the natural functional impairment tends to impart harm rather than benefit in geriatrics $[3,4]$.

Drug-drug interaction is the modification, increase or decrease in the effects of drugs when simultaneously administered with another drug. This leads to severe adverse effects which are totally preventable in most cases if suitably managed [5]. Geriatric population is at a higher risk to these interactions due to the natural functional impairment, and identification of the drug-drug interactions in this population becomes imperative $[6,7]$. 
In Pakistan, monitoring of pharmacotherapy in geriatrics in neglected. Moreover, the prevalence of drug-drug interactions is also not known. Thus, understanding the mechanisms and factors involved in these potential drug-drug interactions (PDDIs) is important to aid the prevention of adverse effects of the interacting drug pairs.

\section{Methods}

The multicentered cross sectional study was conducted at the critical care units of four tertiary care hospitals in Peshawar, Pakistan; Lady Reading Hospital (LRH), Khyber Teaching Hospital (KTH), Hayatabad Medical Complex (HMC) and Northwest General Hospital and Research Center (NWGH \& RC). The former 3 hospitals are government run, while the latter is a private hospital. The critical care units included were Medical Intensive Care Unit (MICU), Surgical Intensive Care Unit (SICU), Cardiac Care Unit (CCU), and Stroke Unit (SU). Patients from the Northwestern region of Pakistan and Afghanistan avail the medical facilities in these hospitals. Patients who fulfilled the inclusion criteria were randomly selected from these critical units. Inclusion criteria was set as patients of age of 60 years or above, prescribed 2 or more drugs, and admitted to the critical care unit for more than $24 \mathrm{~h}$. Data of 2960 patients was collected over the period of 1 year (Dec 2013 - Dec 2014) of which 1044 met the inclusion criteria.

Prior to collection of data, ethical approvals from the respective hospitals were obtained beforehand vide letter number 8075-79/HMC, 488/pharm (KTH), 010 (LRH), and NWGH/Research/01. A predesigned proforma was used to collect the patient demographic data (age, gender, hospital, unit, date of admission and discharge) and treatment profile (diagnosis, drugs administered, dose and frequency, duration of drug administration); patient identification and other personal data were not disclosed. ICH guidelines for good clinical practice were followed [8]. Written informed consent was not necessary because no personal patient data has been included in the manuscript and data was collected from the medication charts of the patients, for which the hospital ethical committee provided approval.

Evaluation of drug-drug interactions were carried out through Micromedex DrugReax [9] which provides details on the severity, documentation, onset and mechanism of the PDDIs. Severity is classified by Micromedex as major, moderate and minor, while documentation is classified as excellent, good and fair. Micromedex also elaborates the mechanism of the interacting drug pairs. Drugs administered simultaneously during treatment were evaluated for PDDIs.
Statistical analysis was performed using IBM SPSS Statistics for Windows, Version 20 (Armonk, NY: IBM Corp.) [10]. Various statistical tools were used for analysis of descriptive data, along with logistic regression to evaluate the association of PDDIs with predictive factors. One-way ANOVA was also employed to observe the difference in means of PDDIs among the four hospitals.

\section{Results}

Of the total 1044 patients included in the study, 877 $(84 \%)$ aged $\leq 75$ years while $167(16 \%)$ patients aged $>75$ years. Male patients were predominant $(60.3 \%)$ as compared to female patients (39.7\%). The mean stay of the patients in critical care units was $4.56 \pm(3.12)$ days, while the mean number of prescribed drugs was 5.99 \pm (1.88) drugs. CCU saw the highest flow of inpatients as compared to other units. Similarly, patients with a primary diagnosis of myocardial infarction were predominant, as shown in Table 1.

Prevalence of potential drug-drug interactions (PDDIs) was $71.07 \%$ of all the patients. A total of 137 (13.1\%) patients were observed to have at least one PDDI, 110 (10.5\%) had 5 PDDIs, while $2(0.2 \%)$ had 19 PDDIs. PDDIs were most prevalent in patients with myocardial infarction, as shown in Table 2.

A total of 3019 PDDIs were observed in 1044 patients, of which 1398 (46.3\%) were of major severity, 1533 (50.8\%) were of moderate severity, 82 (2.7\%) were of minor severity while $6(0.2 \%)$ PDDIs were contraindicated. In terms of documentation, 372 (12.3\%) PDDIs were of excellent, 1485 (49.2\%) PDDIs were of good, and 1162 (38.5\%) PDDIs were of fair documentation. The onset of 1758 (58.2\%) PDDIs was unknown, while $529(17.5 \%)$ and 732 (24.2\%) PDDIs were of rapid and delayed onset respectively. Pharmacodynamic nature PDDIs (66.5\%) were common, while synergistic mechanism (44\%) was predominantly involved in the PDDIs.

Multivariate logistic regression was applied to associate multiple predictors with the presence of PDDIs. whereas, the individual effect of predictors was also evaluated by applying univariate logistic regression.

Univariate logistic regression revealed a positive, statistically significant association between the presence of PDDIs with the following independent variables: $>6$ prescribed drugs, $>3$ days stay in the critical care unit and diagnosis of myocardial infarction, cerebrovascular accident and acute coronary syndrome.

Multivariate logistic regression analysis revealed that the presence of PDDIs was 2.8 times more likely in patients prescribed $>6$ drugs, and 0.5 times more likely in patients of age $>75$ years. The results of univariate and multivariate logistic regression are shown in Table 3.

A total of 225 drug combinations were involved in all the PDDIs, of which $70.2 \%$ attributed to 16 pairs. The 
Table 1 Demographics and general characteristics of study population ( $N=1044)$

\begin{tabular}{|c|c|c|c|}
\hline Variables & Mean \pm SD & Frequency (\%) & Range \\
\hline Gender & - & & - \\
\hline Male & - & $630(60.3 \%)$ & - \\
\hline Female & - & $414(39.7 \%)$ & - \\
\hline Age (years) & $68.53( \pm 7.81)$ & - & $60-100$ \\
\hline Drugs prescribed per patient & $5.99 \pm(1.88)$ & - & $2-13$ \\
\hline Stay in ICU (days) & $4.56 \pm(3.12)$ & - & $1-38$ \\
\hline Critical Unit & - & $1044(100 \%)$ & - \\
\hline Surgical ICU & - & $151(14.5 \%)$ & - \\
\hline Medical ICU & - & $264(25.3 \%)$ & - \\
\hline Cardiac ICU & - & $499(47.7 \%)$ & - \\
\hline Stroke Unit & - & $130(12.5 \%)$ & - \\
\hline Hospital & - & $1044(100 \%)$ & - \\
\hline NWGH & - & $420(40.2 \%)$ & - \\
\hline $\mathrm{LRH}$ & - & $174(16.7 \%)$ & - \\
\hline KTH & - & $223(21.4 \%)$ & - \\
\hline $\mathrm{HMC}$ & - & $227(21.7 \%)$ & - \\
\hline Diagnosis & - & $1044(100 \%)$ & - \\
\hline Myocardial Infarction & - & $261(25.00 \%)$ & - \\
\hline Cerebrovascular Accident & - & 96 (9.20\%) & - \\
\hline Acute Coronary Syndrome & - & $82(7.85 \%)$ & - \\
\hline Heart Failure & - & $59(5.65 \%)$ & - \\
\hline Chronic Obstructive Pulmonary Disorder & - & $34(3.26 \%)$ & - \\
\hline Miscellaneous & - & $512(49.04 \%)$ & - \\
\hline
\end{tabular}

ICU Intensive care unit, NWGH Northwest General Hospital, LRH Lady Reading Hospital, KTH Khyber Teaching Hospital, HMC Hayatabad Medical Complex, SD Standard Deviation

common interacting pairs along with their potential outcomes and management are shown in Table 4.

One-way ANOVA revealed that there was a significant difference $(p<0.001)$ in the means of PDDIs among all the hospitals. Post hoc test showed that there was a significant difference $(p<0.05)$ in the means of PDDIs between NWGH and the rest of the hospitals. Moreover, there was a significant difference $(p<0.001)$ in the means of PDDIs among all the units. Post hoc test showed that there was a significant difference $(p<0.05)$ in the means of PDDIs between CCU and the rest of the units and between SU and the rest of the units.

\section{Discussion}

Previously neglected, the prevalence of PDDIs and their potential outcomes in geriatrics were studied for the first time in Pakistan. The present study revealed a high prevalence of PDDIs in the elderly population. This coincides with the results of other studies conducted in geriatrics and patients admitted to other critical care units $[3,11]$. The prevalence of PDDIs was higher in NWGH, which is a private hospital as compared to the other three hospitals. This variation in prevalence may be due to the higher number of prescribed drugs per patient in NWGH. PDDIs is of great concern in elderly population, due to the physiological changes that occur with aging, which may lead to an increased risk of adverse effects due to drug-drug interactions.

The risk of cardiovascular diseases increases with age [12], and the current study also reported cardiovascular disorders to be the most prevalent in the study population. Cardiovascular disorders were also significantly associated with the presence of PDDIs. This emphasizes for greater care when dealing with geriatrics with a cardiovascular disorder.

PDDIs of major and moderate severity were prevalent. A limited number of other studies have observed the categories of PDDIs in elderly. Studies conducted in elderly population at tertiary hospitals reported PDDIs of moderate severity to be the most prevalent $[3,13]$. Another study conducted in geriatrics in outpatient settings reported that most of the patients had PDDIs of major severity [14]. Similarly, studies conducted in ICU's also reported moderate and major severity PDDIs to be 
Table 2 Characteristics of potential drug-drug interactions

\begin{tabular}{ll}
\hline Variables & No. of Patients (\%) \\
\hline Potential drug-drug interactions & $742(71.07 \%)$ \\
Present & $302(28.93 \%)$ \\
Absent & \\
Most severe PDDI seen in each patient & $6(0.81 \%)$ \\
Contraindicated & $618(83.29 \%)$ \\
Major & $113(15.23 \%)$ \\
Moderate & $5(0.67 \%)$ \\
Minor & \\
Diseases with highest prevalence of PDDIs & $257(34.64 \%)$ \\
Myocardial Infarction & $82(11.05 \%)$ \\
Cerebrovascular Accident & $79(10.65 \%)$ \\
Acute Coronary Syndrome & $44(5.93 \%)$ \\
Heart Failure &
\end{tabular}

among the most prevalent $[15,16]$. PDDIs of pharmacodynamic nature were prevalent in the present study due to the involvement of cardiovascular drugs, the mechanism of interaction of most of them was synergistic or antagonistic.

Furthermore, a significant relationship was observed between PDDIs with polypharmacy and age. A cross-sectional study conducted in Brazil also reported a similar significant association between PDDIs with polypharmacy and age [17]. Another research observed a strong association between polypharmacy and negative clinical consequences in elderly population [18]. A Swedish study also reported a similar relationship between drugdrug interactions and increasing number of prescribed drugs [19]. A US study also found a significant association between PDDIs and polypharmacy [20].

Aspirin was involved in most of the prevalent PDDIs. This drug is one of the most common drug used in cardiovascular disorders, however due to its potential for an interaction with other drugs, its use must be continuously monitored for any adverse effects. The frequent use of aspirin and its potential for involvement in PDDIs has also been reported by Tushar et al. in geriatric outpatients [14].

\section{Limitations}

The current study design could not measure the actual adverse clinical outcomes of the PDDIs, for which further studies have to be conducted. Furthermore, these results are specific for geriatrics so must be generalized with caution in pediatric and adult population.

\section{Conclusion}

A higher prevalence of PDDIs in geriatrics poses a great health concern, due to the weak physiological condition of the aging population. Thus, avoidance of PDDIs and managing them appropriately becomes vital. Monitoring systems should be placed in developing countries to monitor not only PDDIs but also other drug related

Table 3 Factors associated with drug-drug interaction using logistic regression $(n=1044)$

\begin{tabular}{|c|c|c|c|c|}
\hline \multirow[t]{2}{*}{ Variable } & \multicolumn{2}{|l|}{ Univariate Regression } & \multicolumn{2}{|c|}{ Multivariate Regression } \\
\hline & OR $(95 \% \mathrm{Cl})$ & $p$-value & OR $(95 \% \mathrm{Cl})$ & $p$-value \\
\hline \multicolumn{5}{|l|}{ Prescribed drugs } \\
\hline$\leq 6$ & Reference & & Reference & \\
\hline$>6$ & $2.172(1.590-2.968)$ & $<0.001$ & $2.870(2.011-4.095)$ & $<0.001$ \\
\hline \multicolumn{5}{|l|}{ Age } \\
\hline$\leq 75$ & Reference & 0.106 & Reference & \\
\hline$>75$ & $0.748(0.526-1.064)$ & & $0.588(0.386-0.897)$ & $<0.05$ \\
\hline \multicolumn{5}{|l|}{ Duration of stay } \\
\hline$\leq 3$ & Reference & $<0.001$ & Reference & \\
\hline$>3$ & $0.622(0.472-0.820)$ & & $0.868(0.626-1.204)$ & 0.397 \\
\hline \multicolumn{5}{|l|}{ Gender } \\
\hline Male & Reference & 0.250 & Reference & \\
\hline Female & $0.853(0.650-1.119)$ & & $0.820(0.595-1.129)$ & 0.224 \\
\hline \multicolumn{5}{|l|}{ Chronic illness } \\
\hline Myocardial infarction & $0.018(0.005-0.058)$ & $<0.001$ & $0.012(0.003-0.040)$ & $<0.001$ \\
\hline Cerebrovascular accident & $0.192(0.080-0.463)$ & $<0.001$ & $0.147(0.059-0.367)$ & $<0.001$ \\
\hline Acute coronary syndrome & $0.043(0.011-0.162)$ & $<0.001$ & $0.037(0.009-0.142)$ & $<0.001$ \\
\hline Heart failure & $0.384(0.157-0.936)$ & $<0.05$ & $0.265(0.105-0.673)$ & $<0.05$ \\
\hline
\end{tabular}




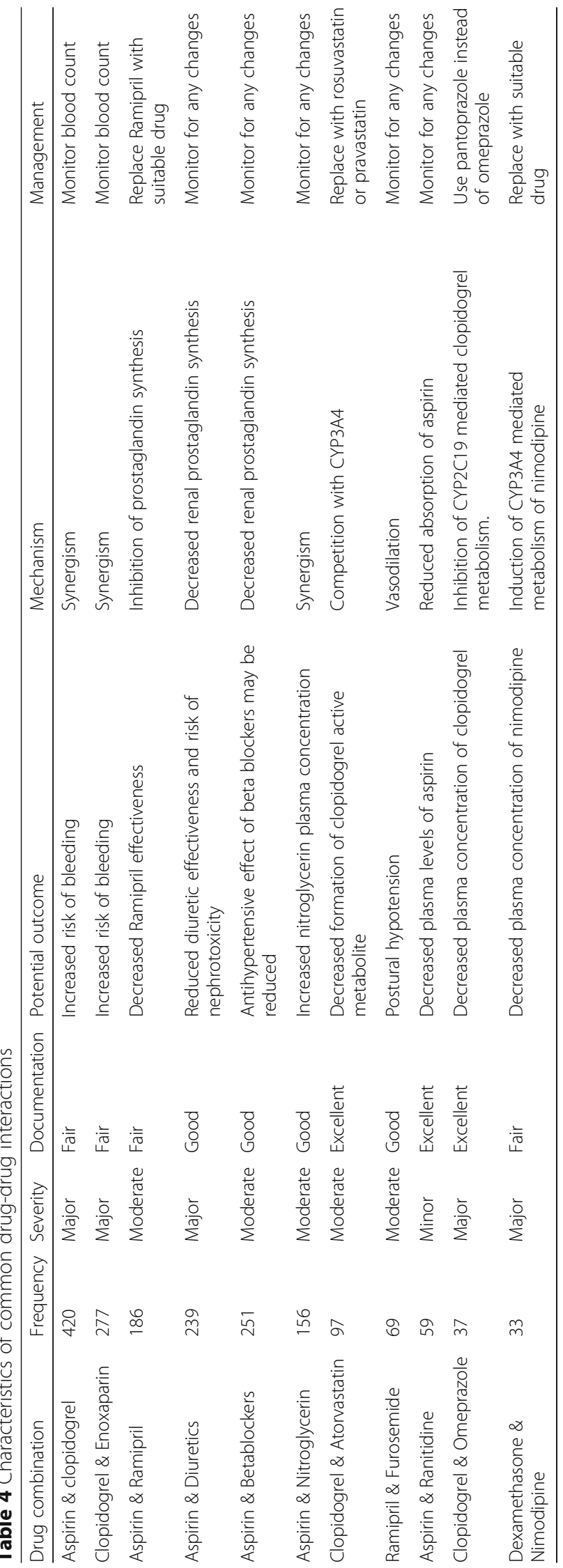


problems to provide quality health care to patients. Moreover, replacing the drugs involved in PDDIs with appropriate drugs having a lesser potential for PDDI can be implemented to further reduce the risk of PDDIs. Education and training regarding this must be provided to the health care professionals.

\section{Additional file}

Additional file 1: Geriatric patient data. This file contains the demographic data and medication profile collected from the treatment charts of the patients. (XLS $3520 \mathrm{~kb}$ )

\section{Abbreviations}

CCU: Cardiac care unit; HMC: Hayatabad medical complex; KTH: Khyber teaching hospital; LRH: Lady reading hospital; MICU: Medical intensive care unit; NWGH \& RC: Northwest general hospital and research center; PDDls: Potential drug-drug interactions; SICU: Surgical intensive care unit; SU: Stroke Unit

\section{Acknowledgments}

The authors are grateful for the support and co-operation provided by the staff of all the hospitals involved in this study.

\section{Funding}

No external funding was provided to the investigators, and was completely self-funded.

\section{Availability of data and materials}

The dataset generated and analyzed during the study is included as Additional file 1.

\section{Authors' contributions}

FS was involved in the concept and design of the study, data collection, analysis and drafting of the article. MA as involved in data collection and analysis. AFK was involved in the drafting and review of the article. TNK was involved in the drafting of the article. SK was involved in the concept and analysis. All authors read and approved the final manuscript.

\section{Ethics approval and consent to participate}

Written informed consent was not necessary because no personal patient data has been included in the manuscript and data was collected from the medication charts of the patients, for which the hospital ethical committee provided approval.

Ethical approvals to collect data from medication charts of the patients were obtained from the respective hospitals, namely ethical committee of Hayatabad medical complex, medical superintendent Khyber teaching hospital, institutional research and ethical board, post graduate medical institute lady reading hospital and ethical committee of northwest general hospital and research center vide letter numbers 8075-79/HMC, 488/pharm (KTH), 010 (LRH), and NWGH/Research/ 01 respectively.

\section{Consent for publication}

Not applicable.

\section{Competing interests}

The authors declare that they have no competing interests.

\section{Publisher's Note}

Springer Nature remains neutral with regard to jurisdictional claims in published maps and institutional affiliations.

\section{Author details}

'Department of Pharmacy, Sarhad University of Science and IT, Peshawar, Pakistan. ${ }^{2}$ University of Vermont Medical Centre, Burlington, VT, USA. ${ }^{3}$ Punjabi Community Health Services, Calgary, Alberta, Canada. ${ }^{4}$ Department of Pharmacy, University of Malakand, Lower Dir District, Pakistan.
Received: 23 August 2018 Accepted: 26 November 2018

Published online: 10 December 2018

\section{References}

1. de Figueiredo TP, de Souza Groia RC, Barroso SCC, do Nascimento MMG, Reis AMM. Factors associated with adverse drug reactions in older inpatients in teaching hospital. Int J Clin Pharm. 2017:39(4):679-85.

2. Pantuzza $L L$, Ceccato $M G B$, Silveira $M R$, Junqueira $L M R$, Reis $A M M$. Association between medication regimen complexity and pharmacotherapy adherence: a systematic review. Eur J Clin Pharmacol. 2017;73(11):1475-89.

3. Salwe KJ, Kalyansundaram D, Bahurupi Y. A study on polypharmacy and potential drug-drug interactions among elderly patients admitted in Department of Medicine of a tertiary care hospital in Puducherry. J Clin Diagn Res. 2016;10(2):FC06-10.

4. Hines LE, Murphy JE. Potentially harmful drug-drug interactions in the elderly: a review. Am J Geriatr Pharmacother. 2011;9(6):364-77.

5. Dookeeram D, Bidaisee S, Paul JF, Nunes P, Robertson P, Maharaj VR, et al. Polypharmacy and potential drug-drug interactions in emergency department patients in the Caribbean. Int J Clin Pharm. 2017:39(5):1119-27.

6. Lin C-F, Wang C-Y, Bai C-H. Polypharmacy, aging and potential drug-drug interactions in outpatients in Taiwan. Drugs Aging. 2011;28(3):219-25.

7. Hohl CM, Dankoff J, Colacone A, Afilalo M. Polypharmacy, adverse drugrelated events, and potential adverse drug interactions in elderly patients presenting to an emergency department. Ann Emerg Med. 2001;38(6):666-71.

8. Bhuiyan P, Rege N. ICH Harmonised tripartite guideline: guideline for good clinical practice; 2001.

9. Micromedex T. Drug-Reax ${ }^{\circledast}$ system. www.micromedexsolutions.com.

10. SPSS I. IBM SPSS statistics for windows, version 20.0. New York: IBM Corp; 2011

11. Uijtendaal EV, Harssel LL, Hugenholtz GW, Kuck EM, Zwart-van Rijkom JE, Cremer OL, et al. Analysis of potential drug-drug interactions in medical intensive care unit patients. Pharmacotherapy. 2014;34(3):213-9.

12. Conroy R, Pyörälä K, Ae F, Sans S, Menotti A, De Backer G, et al. Estimation of ten-year risk of fatal cardiovascular disease in Europe: the SCORE project. Eur Heart J. 2003;24(11):987-1003

13. Nobili A, Pasina L, Tettamanti M, Lucca U, Riva E, Marzona I, Monesi L, Cucchiani R, Bortolotti A, Fortino I, Merlino L, Walter Locatelli G, Giuliani G. Potentially severe drug interactions in elderly outpatients: results of an observational study of an administrative prescription database. J Clin Pharm Ther. 2009;34(4):377-386

14. Nishandar TB, Kale AS, Pise HN. Study of potential drug interactions between prescribed drugs in geriatric patients attending outpatien department in a government tertiary care hospital in Maharashtra. Int J Basic Clin Pharmacol. 2016;5(4):1569-73

15. Shakeel F, Khan JA, Aamir M, Asim SM, Ullah I. A multicentered pharmacoepidemiological approach to evaluate clinically significant potential drug-drug interactions in medical intensive care settings in Pakistan. Hong Kong J Emerg Med. 2018;25(4):190-5.

16. Shakeel F, Khan JA, Aamir M. Relationship of factors affecting clinically important drug interactions and their significance in surgical intensive care units in Pakistan. Lat Am J Pharm. 2018;37(4):643-50.

17. Novaes PH, da Cruz DT, Lucchetti ALG, Leite ICG, Lucchetti G. The "iatrogenic triad": polypharmacy, drug-drug interactions, and potentially inappropriate medications in older adults. Int J Clin Pharm. 2017:39(4): $818-25$

18. Maher RL, Hanlon J, Hajjar ER. Clinical consequences of polypharmacy in elderly. Expert Opin Drug Saf. 2014;13(1):57-65

19. Johnell K, Klarin I. The relationship between number of drugs and potential drug-drug interactions in the elderly. Drug Saf. 2007;30(10):911-8.

20. Greene M, Steinman MA, McNicholl IR, Valcour V. Polypharmacy, drugdrug interactions, and potentially inappropriate medications in older adults with human immunodeficiency virus infection. J Am Geriatr Soc. 2014:62(3):447-53. 